Washington University School of Medicine Digital Commons@Becker

Open Access Publications

3-1-2021

\title{
Uveal melanoma cells use ameboid and mesenchymal mechanisms of cell motility crossing the endothelium
}

\author{
Michael D. Onken \\ Washington University School of Medicine in St. Louis \\ Kendall J. Blumer \\ Washington University School of Medicine in St. Louis \\ John A. Cooper \\ Washington University School of Medicine in St. Louis
}

Follow this and additional works at: https://digitalcommons.wustl.edu/open_access_pubs

Please let us know how this document benefits you.

\section{Recommended Citation}

Onken, Michael D.; Blumer, Kendall J.; and Cooper, John A., "Uveal melanoma cells use ameboid and mesenchymal mechanisms of cell motility crossing the endothelium." Molecular Biology of the Cell. 32, 5. 413 - 421. (2021).

https://digitalcommons.wustl.edu/open_access_pubs/10133

This Open Access Publication is brought to you for free and open access by Digital Commons@Becker. It has been accepted for inclusion in Open Access Publications by an authorized administrator of Digital Commons@Becker. For more information, please contact vanam@wustl.edu. 


\title{
Uveal melanoma cells use ameboid and mesenchymal mechanisms of cell motility crossing the endothelium
}

\author{
Michael D. Onken ${ }^{a, *}$, Kendall J. Blumerb, and John A. Coopera \\ aDepartment of Biochemistry and Molecular Biophysics and bepartment of Cell Biology \& Physiology, Washington \\ University School of Medicine, Saint Louis, MO 63110
}

\begin{abstract}
Uveal melanomas (UMs) are malignant cancers arising from the pigmented layers of the eye. UM cells spread through the bloodstream, and circulating UM cells are detectable in patients before metastases appear. Extravasation of UM cells is necessary for formation of metastases, and transendothelial migration (TEM) is a key step in extravasation. UM cells execute TEM via a stepwise process involving the actin-based processes of ameboid blebbing and mesenchymal lamellipodial protrusion. UM cancers are driven by oncogenic mutations that activate $\mathrm{G} \alpha \mathrm{q} / 11$, and this activates TRIO, a guanine nucleotide exchange factor for RhoA and Rac1. We found that pharmacologic inhibition of G $\alpha q / 11$ in UM cells reduced TEM. Inhibition of the RhoA pathway blocked amoeboid motility but led to enhanced TEM; in contrast, inhibition of the Rac1 pathway decreased mesenchymal motility and reduced TEM. Inhibition of Arp2/3 complex allowed cells to transmigrate without intercalation, a direct mechanism similar to the one often displayed by immune cells. BAP1-deficient (+/-) UM subclones displayed motility behavior and increased levels of TEM, similar to the effects of RhoA inhibitors. We conclude that RhoA and Rac1 signaling pathways, downstream of oncogenic G $\alpha q / 11$, combine with pathways regulated by BAP1 to control the motility and transmigration of UM cells.
\end{abstract}

Monitoring Editor

Valerie Marie Weaver

University of California,

San Francisco

Received: Apr 14, 2020

Revised: Dec 17, 2020

Accepted: Dec 30, 2020

\section{INTRODUCTION}

Uveal melanoma (UM) is a highly aggressive cancer arising in the uveal layers of the eye, including the choroid, ciliary bodies, and iris. The eye is the second most common site of melanoma. UM and cutaneous melanoma are distinct diseases (van der Kooij et al., 2019; Urtatiz et al., 2020). Oncogenesis of UM tumors is driven by constitutive activation of the $\mathrm{Gq}$ family of alpha subunits, $\mathrm{G} \alpha q$ and Ga11 (GNAQ and GNA11) in over $90 \%$ of tumors, while cutaneous melanomas are driven by BRAF and NRAS mutations (Van Raamsdonk et al., 2009, 2010). Ten-year survival of patients with primary UM tumors is about $50 \%$, compared with over $90 \%$ for cutaneous melanoma (Carvajal et al., 2017). Despite local control of the

This article was published online ahead of print in MBoC in Press (http://www .molbiolcell.org/cgi/doi/10.1091/mbc.E20-04-0241) on January 6, 2021.

*Address correspondence to: Michael D. Onken, (mdonken@wustl.edu).

Abbreviations used: TEM, transendothelial migration; UM, uveal melanoma. (C) 2021 Onken et al. This article is distributed by The American Society for Cell Biology under license from the author(s). Two months after publication it is available to the public under an Attribution-Noncommercial-Share Alike 3.0 Unported Creative Commons License (http://creativecommons.org/licenses/by-nc-sa/3.0).

"ASCB®," "The American Society for Cell Biology®," and "Molecular Biology of the Cell $($ " are registered trademarks of The American Society for Cell Biology. primary eye tumor being achieved in over $95 \%$ of patients, UM has a high rate of metastasis and lethal outcome (Krantz et al., 2017). The anatomy of the eye essentially prevents local spread, and the posterior chamber of the eye lacks lymphatic vessels, so metastatic spread of UM to distant organs occurs only through the bloodstream (Clarijs et al., 2001).

Hematogenous spread of UM cells begins with shedding of cells from the primary eye tumor. The tumor vasculature of UM is irregular and discontinuous, providing a poor barrier to cell shedding (Clarijs et al., 2002; Mihic-Probst et al., 2012). As a result, circulating tumor cells are found in all patients, even those with low-risk tumors that do not develop metastatic disease (Keilholz et al., 2004; Callejo et al., 2006; Anand et al., 2019). Because shedding of cells into the blood is common, extravasation of circulating tumor cells out of blood vessels at distant sites may be a key rate-limiting step for UM metastasis.

To investigate transendothelial migration (TEM), we developed a cell culture system employing primary human endothelial monolayers grown on polyacrylamide hydrogels, and we followed TEM in real time with living cells (Onken et al., 2014b). Using this approach, we discovered that the migration of UM cells occurs via a stepwise 
process (Onken et al., 2014a). Suspended UM cells attach to endothelial cells and then intercalate between adjacent endothelial cells, flattening into the monolayer. UM cells remain intercalated for up to several hours before releasing from the endothelial monolayer and migrating underneath it. The cellular and molecular mechanisms driving each step have not been characterized and may provide information for preventing circulating tumor cells from exiting the vasculature.

Cell migration and the actin cytoskeleton are key features of UM metastasis. Spread of UM tumors to distant organs is known to be promoted by loss of BAP1, a chromatin remodeling factor (Harbour et al., 2010), and we found that depletion of BAP1 increases the overall rate of TEM in our system (Onken et al., 2014a). BAP1 is a histone deubiquitinase recruited to promoters of genes (Yen et al., 2018), and we found that several actin cytoskeleton regulator genes are targeted by BAP1 in UM cells (Yen et al., 2018). In parallel, constitutively active $\mathrm{Gq} / 11$, the oncogenic driver of UM, activates the dual nucleotide exchange factor TRIO, which in turn activates the Rho-like small GTPases RhoA and Rac1 (Schmidt and Debant, 2014) that regulate the actin cytoskeleton during cell migration (Feng et al., 2014, 2019). We hypothesize that regulation of actin-based processes, downstream of BAP1 and $\mathrm{Gq} / 11$, and via the RhoA and Rac1 pathways, controls the migration of UM cells.

Rac1 drives actin polymerization at the leading edge of a migrating cell and promotes focal complex assembly (Nobes and Hall, 1995; Parri and Chiarugi, 2010), resulting in the formation of lamellae and lamellipodia, which move the cell forward (Nobes and Hall, 1995, 1999; Parri and Chiarugi, 2010). Cell movement based on Rac1-driven lamellipodial protrusions is often referred to as "mesenchymal" motility (Cooper et al., 2015; Byrne et al., 2016). Rac1 activates downstream effectors, including WAVE2, which activate Arp2/3 complex for branched actin polymerization (Haga and Ridley, 2016). Rac1 also activates p21-activated kinase (PAK), which has several important substrates (Bokoch, 2003; Zhao and Manser, 2012), including GEF-H1, a regulator of RhoA (Guilluy, Garcia-Mata, and Burridge, 2011).

RhoA activates Rho-associated protein kinases 1 and 2 (ROCK1 and ROCK2) (Sanz-Moreno et al., 2008; Acton et al., 2014), which increase actomyosin contractility via myosin light-chain (MLC) phosphorylation. This results in increased cortical contractile forces, which enhance membrane blebbing (Chrzanowska-Wodnicka and Burridge, 1996). Cell movement based on RhoA-driven contractile blebbing is often referred to as "amoeboid" motility (Cooper et al., 2015; Byrne et al., 2016). Biochemically, the RhoA and Rac1 pathways can be antagonistic, with opposing roles in cell migration (Sanz-Moreno et al., 2008; Guilluy et al., 2011). ROCK signaling can antagonize Rac1 via ARHGAP22, and Rac1WAVE2 activity can antagonize ROCK signaling (Sanz-Moreno et al., 2008). Interacting and antagonistic pathways downstream of RhoA and Rac1 control switching between amoeboid and mesenchymal migration and have been identified in 2D and 3D culture conditions in normal fibroblasts and melanoma and breast cancer cells (Cooper et al., 2015; Sander et al., 1999; Byrne et al., 2016).

Here, we investigated the roles of these signaling pathways with a combination of pharmacologic and molecular genetic perturbations, using direct live-cell observation of TEM. We found that UM cells in suspension show predominantly amoeboid motility driven by the RhoA pathway and that UM cells switch from amoeboid motility to mesenchymal motility as they adhere to and migrate through the endothelial monolayer.

\section{RESULTS}

First, we established methods to quantify UM cell behavior during TEM, allowing us to measure the effects of pharmacologic and genetic perturbations. We characterized the movements of two UM cell lines, 92.1 and Mel202, before and during TEM from live-cell movies (Figure 1A and Supplemental Video S1). UM cells displayed amoeboid blebbing while in suspension, immediately after addition to endothelial monolayers, and for a short while after coming to rest on the monolayers. The cells continued blebbing during their initial interactions with the endothelial monolayer. Next, they stopped blebbing and formed lamellipodial protrusions. The cells proceeded to spread, flatten, and intercalate themselves between endothelial cells. The steps of adhesion and intercalation occurred relatively quickly and were complete within a few hours. After a longer time, the cells formed new lamellipodial protrusions that extended underneath the endothelial monolayer, and the cells migrated away, in agreement with our previous observations (Onken et al., 2014a).

We quantified the progression of UM cells through each of these steps during TEM (see Figure 1B for an example) by measuring and calculating the following parameters: 1) total number of cells completing each step after $2 \mathrm{~h}$ (Figure 1C), 2) maximum instantaneous rate of cells completing each step (Figure 1D), and 3) time required for half the expected number of cells to complete each step (Figure $1 \mathrm{E})$. The endothelial monolayers were prepared from primary human dermal microvascular endothelial cells (HDMVECs), for which different lots display a certain level of biological variability. To account for this variability among lots, control (DMSO-treated) cell samples were produced with every experiment, and the results from these control samples were used to normalize the results from experimental samples.

\section{Inhibition of the oncogenic driver $\mathrm{Gq} / 11$ blocks transendothelial migration}

Constitutively activating mutations in $\mathrm{Gq} / 11$ are the driving oncogenes for over $90 \%$ of UM tumors (Moore et al., 2016). Gq/11 activates the dual-GEF TRIO, which activates RhoA and Rac1 in UM cells (Feng et al., 2014). We used FR900359, a potent inhibitor of Gq/11 in UM cells (Onken et al., 2018), to block TRIO activation by Gq. UM cells treated with FR900359 for 24 h showed significant decreases in progression for all steps of transmigration (Figure 1A and Supplemental Video S1). Adhesion and intercalation were reduced to $40 \%$ of untreated activity, and neither extension nor migration was detected during any 2 -h experiment (Figure $1 \mathrm{C}$ ). Maximum rates of progression of each step decreased by $>4$-fold (Figure 1D), and time to reach maxima increased by $>2$-fold (Figure 1E). Thus, inhibition of the oncogenic driver mutation blocks most UM cells from transmigrating and slows the progression of the few cells that do transmigrate.

\section{Inhibition of the RhoA pathway increases transendothelial migration}

Activated TRIO regulates both RhoA and Rac1, which control opposing cell morphology pathways (Sanz-Moreno et al., 2008). We interrogated each pathway independently to dissect their regulatory roles in UM cells during TEM. Inhibition of ROCK, the immediate downstream effector of activated RhoA, by Y-27632 blocked the amoeboid blebbing activity of UM cells in suspension and on the surface of the monolayers (Figure 2A and Supplemental Video S2). Surprisingly, UM cells treated with Y-27632 showed significant increases in both the percentage of cells completing each step of TEM (Figure 2B) and the rates of cells progressing through each step (Figure 2, C and D). To confirm that these results were specific to the Rho pathway and not caused by off-target effects of the 


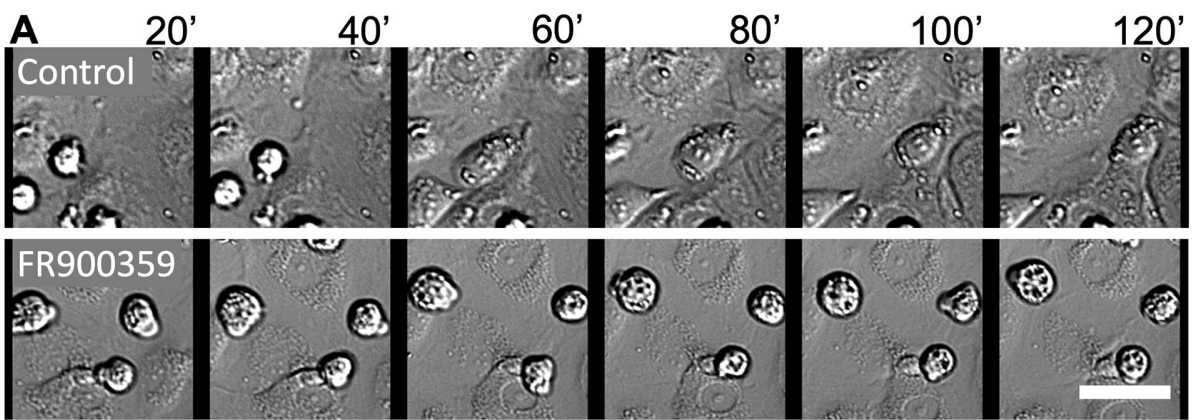

B

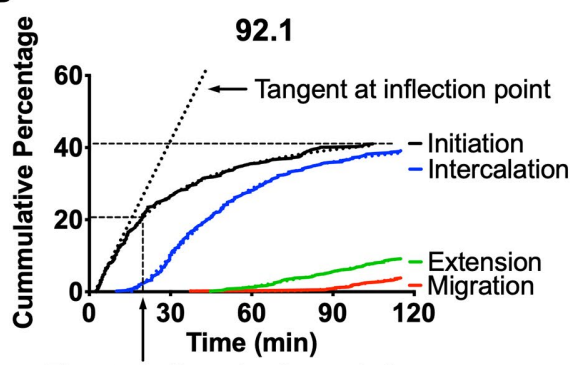

Time to half maximal completion
C
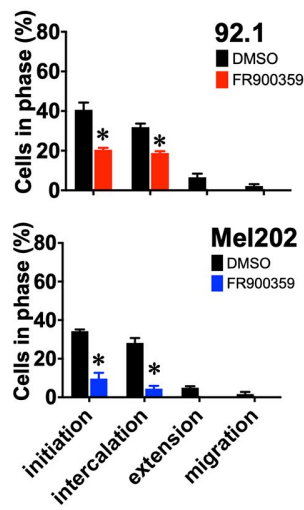

D
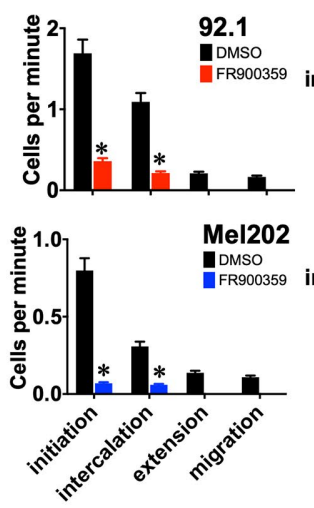

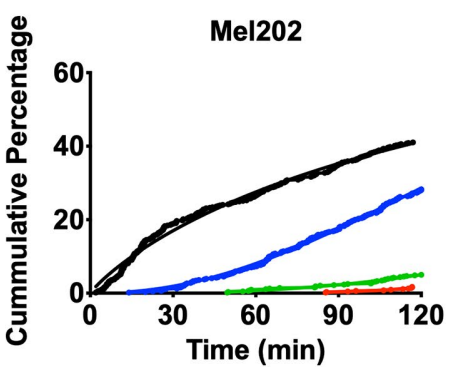

E

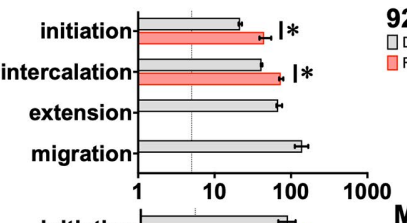

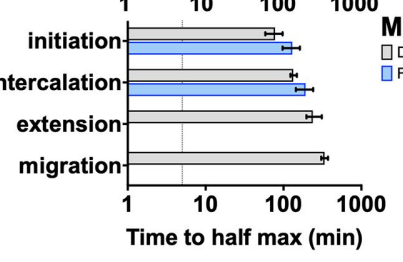

92.1

DMSO TFR900359 Mel202 पDMSO FR900359
FIGURE 1: Quantification of TEM of untreated and FR900359-treated UM cells. (A) Frames from representative movies of TEM by control 92.1 UM cells (upper panels and Supplemental Video S1) and 92.1 cells treated with FR900359 (lower panels), at 20-min intervals. Scale bar $=50 \mu \mathrm{m}$. (B) Cumulative percentage of cells undergoing each step of UM cell transmigration. Results are from three 2-h movies for each condition for each cell line. The total numbers of 92.1 cells scored were 321 (vehicle) and 486 (FR900359); the total numbers of Mel202 cells scored were 365 (vehicle) and 252 (FR900359). Curves from both cell lines under control and treated conditions were quantified to generate the bar graphs in panels $C-E$ ( ${ }^{*} p<0.01$ ). (C) Endpoint values for each step after $2 \mathrm{~h}$. (D) Maximum rate of each step calculated from the slope of the tangent at the inflection point. (E) Time to reach half of the calculated maximum plateau for each step.
Inhibition of the Rac1 pathway blocks transendothelial migration

Actin-based lamellipodial protrusions are a prominent feature of UM cells as the cells intercalate into the endothelial monolayer and then extend below the monolayer and migrate underneath it. We hypothesized that these protrusions were driven by activation of Rac1. To inhibit Rac1, we treated UM cells with NSC-23766, which blocks the activation of Rac1 by exchange factors. The cells showed increased amoeboid blebbing and decreased lamellipodial protrusion, with an overall impairment of TEM (Figure 3 and Supplemental Video S3). The numbers of cells completing each step were significantly reduced, as were the rates of completion within each step (Figure 3, B-D). To confirm that these results were specific to the Rac pathway and not caused by off-target effects of the inhibitor, we expressed a dominant-negative form of Rac1 in both UM cell lines. Mel202 UM cells expressing dominant-negative Rac1 showed significant reduction of all steps of TEM, similar to the effects of NSC23766 treatment. The effect on 92.1 cells was a significant increase in the time for completing each step (Figure 3, B-D). Taken together, these decreases in TEM parameters were similar to the stronger effects observed for cells treated with FR900359 above (Figure 1, C-E).

Rac1 directly activates PAKs, which phosphorylate target proteins (Zhao and Manser, 2012). Treatment of UM cells with the PAK inhibitor IPA-3 enhanced blebbing and reduced TEM (Figure 3, A and B), similarly to NSC-23766. Fewer cells completed each step, and rates of completion within each step were reduced (Figure 3, C-E). Interestingly, UM cells treated with either NSC23766 or IPA-3 also showed larger blebs and increased extracellular debris (Figure 3A and Supplemental Video S3) compared with untreated cells (Figure 1A and Supplemental Video S1), suggesting dysregulation of bleb size and retraction resulting from inhibition of the Rac pathway. inhibitor, we expressed a dominant-negative form of RhoA. For both UM cell lines dominant-negative RhoA expression caused increases in TEM similar to the effects of Y-27632 treatment (Figure 2, B-D).

Downstream of ROCK in the RhoA pathway is nonmuscle myosin II, the motor protein that powers cellular contractility and membrane tension and thus drives amoeboid blebbing (Wyckoff et al., 2006). Inhibition of nonmuscle myosin II in UM cells by treatment with blebbistatin blocked blebbing activity (Figure 2A and Supplemental Video S2) and increased the rate of TEM (Figure 2, B-E). These effects were similar to those of Y-27632. Thus, the effects of ROCK inhibition on UM cells appear to occur through regulation of actomyosin contractility.
Inhibition of Arp2/3 complex: uveal melanoma cells transmigrate and bypass intercalation

The actin-based protrusions that occur during lamellar and lamellipodial cell migration are driven by the rapid formation and growth of branched actin networks at the cell periphery. Actin branching is nucleated by the Arp2/3 complex, which is activated by the WAVE complex, a downstream target of activated Rac1. We used the Arp2/3 inhibitor CK-666 (Nolen et al., 2009) to interrogate the role of branched actin formation in Rac1 regulation of TEM. UM cells treated with CK-666 showed extensive blebbing, similar to the effects of NSC-23766 and IPA-3 (Figure 4A and Video S4). The CK-666-treated cells failed to switch from amoeboid blebbing to 


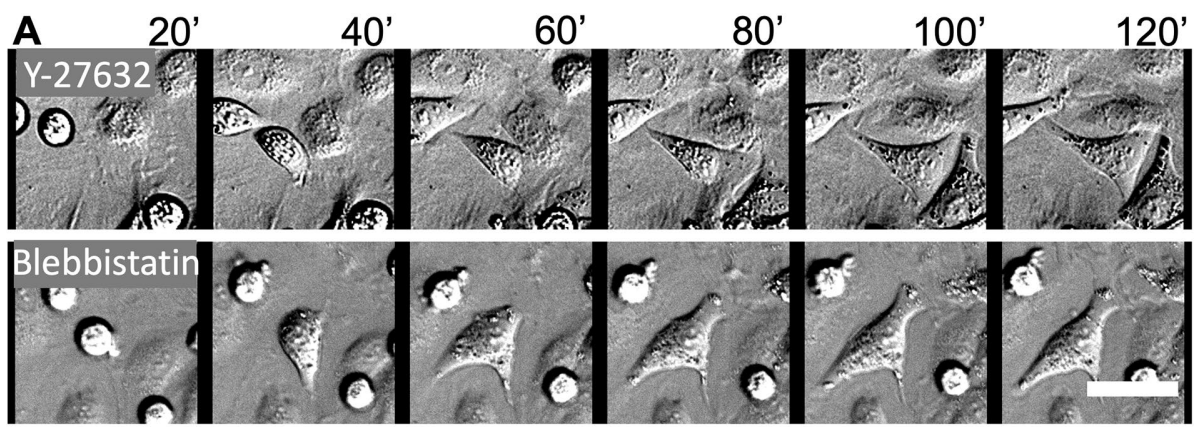

B
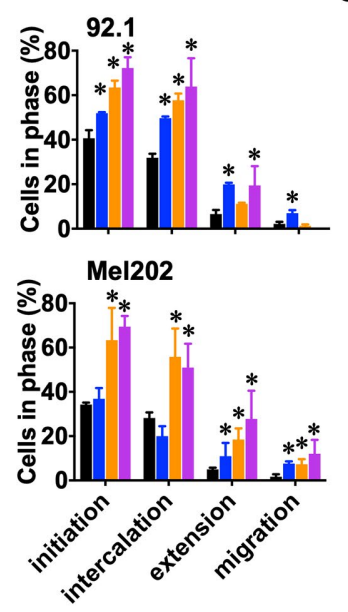

C

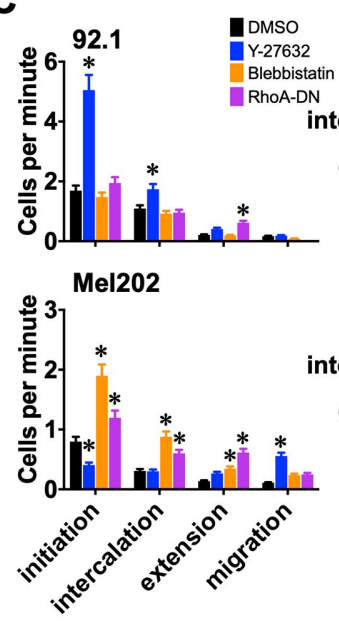

D
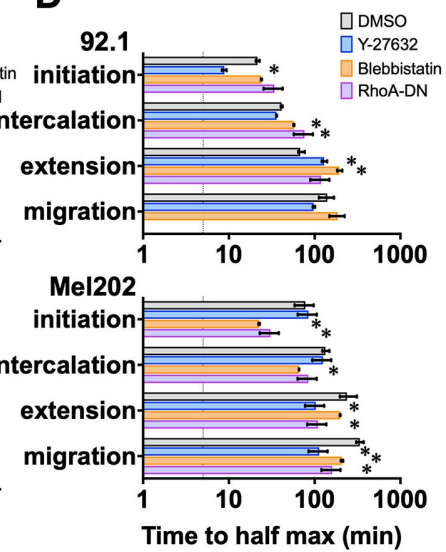

FIGURE 2: Effects of Rho pathway inhibition on TEM of UM cells. (A) Frames from representative movies of TEM by $92.1 \mathrm{UM}$ cells treated with RhoA-dependent ROCK1 inhibitor Y-27632 (upper panels and Supplemental Video S2) or myosin inhibitor blebbistatin (lower panels), at 20-min intervals. (B) Endpoint values of cells that have undergone each step of UM cell transmigration treated with Y-27632 or blebbistatin, or expressing dominant negative RhoA, based on results from three 2-h movies for each condition for each cell line. The total numbers of 92.1 cells scored were 355 (vehicle), 502 (Y-27632), 285 (blebbistatin), and 103 (RhoA-DN); the total numbers of Mel202 cells scored were 569 (vehicle), 172 (Y-27632), 700 (blebbistatin), and 151 (RhoA-DN). (C) Maximum rates for each step of TEM. (D) Times to reach half of the calculated maximum plateaus. Inhibition of the Rho pathway causes more UM cells to transmigrate and at a faster rate. Scale bar, $50 \mu \mathrm{m} ;{ }^{\star} p<0.01$.

mesenchymal motility; however, they proceeded to perform TEM, using a direct manner that bypassed the process of intercalation (Figure 4A and Supplemental Video S4). This TEM migration behavior is similar to that displayed by immune cells, including lymphocytes and NK cells (Onken et al., 2014b).

\section{Depletion of the metastasis suppressor BAP1 increases transendothelial migration}

Loss of the chromatin-remodeling factor BAP1 drives metastasis in UM tumors (Harbour et al., 2010; Matatall et al., 2013). BAP1 is enriched at the transcriptional promoters of TRIO, cortactin, and several GTPase activating proteins (GAPs) and guanine nucleotide exchange factors (GEFs) in UM (Supplemental Figure S1A; Yen et al., 2018), and several of these BAP1 targets are differentially regulated in BAP1-deficient class 2 UM tumors (Supplemental Figure S1B; Onken et al., 2004), which could alter the balance of RhoA and Rac1 pathways. We used CRISPR/Cas9 genome editing to generate BAP1-deficient UM cells. The homozygous loss of BAP1 was lethal in all of several UM cell lines tested, consistent with previous studies (Testa et al., 2011; Dey et al., 2012; Matatall et al., 2013; Hart et al.,
2015). However, we were able to generate two independent BAP1-deficient heterozygous subclones of the 92.1 UM cell line, 92.1-1D7 and 92.1-2D3. Both cell lines displayed amoeboid blebbing while in suspension, and they switched to forming lamellipodial protrusions during intercalation, extension, and migration, similar to parental BAP1(+/+) cells (Figure 5A and Supplemental Video S5). However, in contrast to parental BAP1 (+/+) cells, both BAP1 (+/-) subclones showed quantitative increases in all steps of TEM. More cells completed all steps, and the times for initiation and intercalation were shorter (Figure 5, C-E). Thus, reduction of BAP1 activity produced effects on TEM similar to those resulting from inhibition of the RhoA pathway, as opposed to the effects of inhibition of the Rac1 pathway (Supplemental Figure S2). These results suggest that BAP1 expression favors activation of the RhoA pathway over the Rac1 pathway, such that loss of BAP1 shifts the balance toward Rac1-driven motility and increased TEM.

\section{DISCUSSION}

Uveal melanoma cells switch modes of motility during transendothelial

\section{migration}

UM cells perform TEM by a stereotypical multistep route, which includes intercalation of the UM cell into the endothelial monolayer (Onken et al., 2014a). Here, we discovered that UM cells exhibit both amoeboid and mesenchymal modes of motility as they migrate through the endothelium during TEM and that the switch from amoeboid to mesenchymal motility is an essential element of intercalation. Previous studies reported amoeboid and mesenchymal morphologies for cutaneous melanoma cells attached to substrates with varying levels of adhesivity (Friedl and Wolf, 2003; Sahai and Marshall, 2003; Sanz-Moreno et al., 2008, 2011); those studies did not involve melanoma cells interacting with the endothelium.

We discovered that the oncogenic driver mutation of UM cells is responsible for promoting TEM. Inhibition of constitutively active Gq/11 in UM cells, by the pharmacologic inhibitor FR900359, led to a nearly complete loss of TEM activity in our assay system. In our previous work, FR900359 caused UM cells to stop dividing and to re-differentiate towards their melanocytic state (Onken et al., 2018). Together, the results suggest that FR900359 has promise as a novel therapeutic agent for UM tumors. The inhibition of TEM observed here may be particularly significant because the lethality of UM tumors is due to metastasis through the bloodstream (Anand et al., 2019; Clarijs et al., 2001) and thus requires TEM.

We discovered that the Rho and Rac pathways activated downstream of the oncogenic mutation in UM cells have distinct and complementary roles in the mechanism of transmigration. Inhibiting the Rho pathway blocked amoeboid motility but increased TEM (Supplemental Figure S2), while inhibiting the Rac pathway 

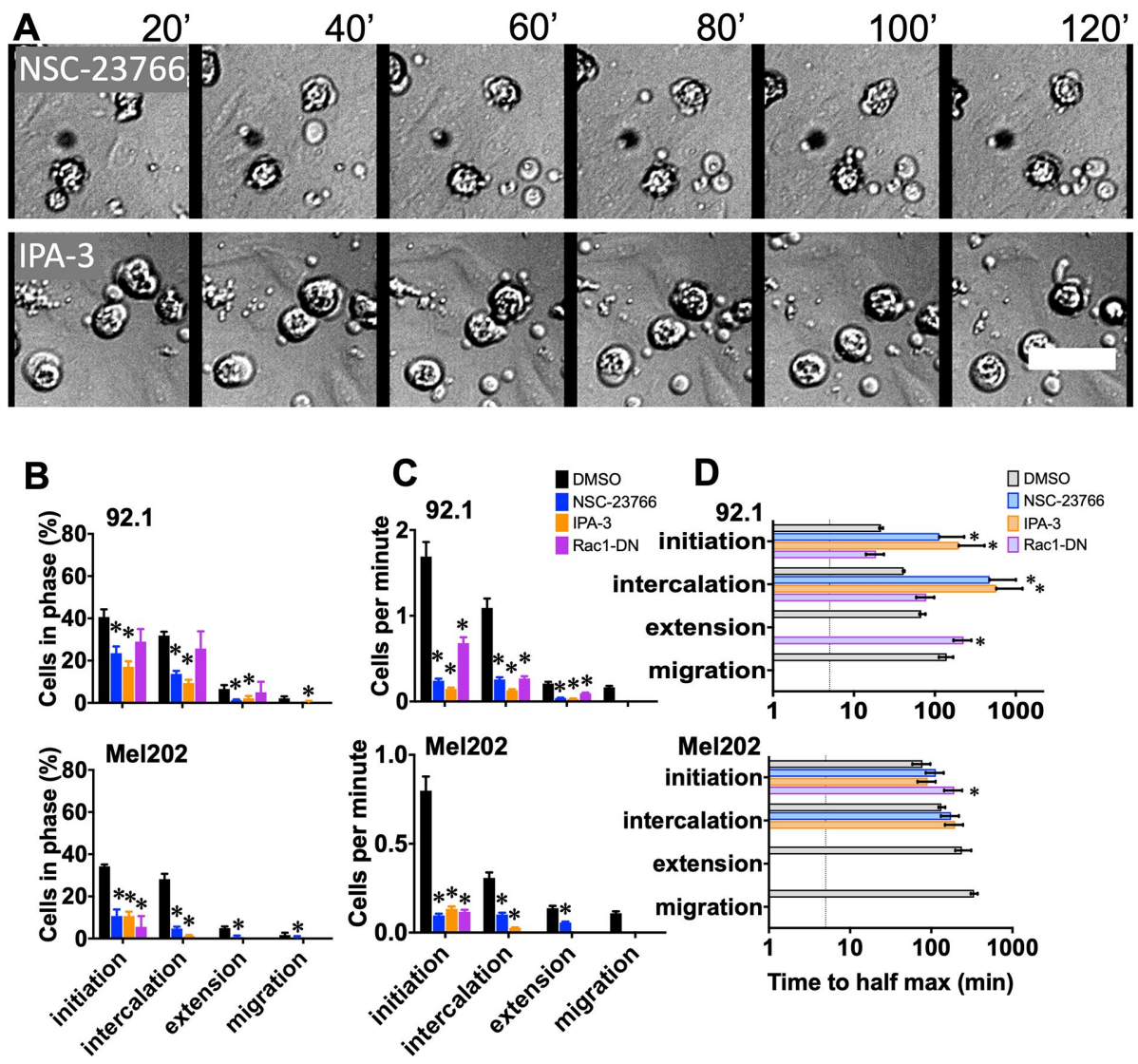

FIGURE 3: Effects of Rac pathway inhibition on TEM of UM cells. (A) Frames from representative movies of TEM by 92.1 UM cells treated with the RacGEF inhibitor NSC-23766 (upper panels and Supplemental Video S3) and the PAK inhibitor IPA-3 (lower panels).

(B) Endpoint values of cells treated with NSC-23766 or IPA-3, or expressing dominant negative Rac1, that have undergone each step based on results from three 2-h movies for each condition for each cell line. The total numbers of 92.1 cells scored were 402 (vehicle), 297 (NSC-23766), 259 (IPA-3), and 138 (Rac1-DN); the total numbers of Mel202 cells scored were 641 (vehicle), 271 (NSC-23766), 417 (IPA-3), and 195 (Rac1-DN). (C) Maximum rates for each step. The number of TEM events was too low to calculate maximum rates of migration. (D) Times to reach half of the calculated maximum plateaus. Inhibition of the Rac pathway caused fewer UM cells to transmigrate and at a slower rate. The number of TEM events was too low to analyze extension and migration accurately. Scale bar, $50 \mu \mathrm{m} ;{ }^{*} p<0.01$.

blocked mesenchymal motility and decreased TEM (Supplemental Figure S2).

We propose the following model of TEM regulation in UM cells, illustrated in Figure 6 and Supplemental Figure S3. First, UM cells display amoeboid morphology and motility as they flow through the bloodstream and make initial contact with the endothelium. This amoeboid behavior depends on active RhoA and actomyosin contraction. Following contact with the endothelium, UM cells convert their morphology and motility to lamellar and lamellipodial protrusions driven by Rac1. These protrusive actions enhance the interactions of UM cells with the endothelial cells, which allows the UM cells to intercalate and flatten into the endothelium and then ultimately leave the endothelium and migrate into surrounding tissues.

\section{Amoeboid and mesenchymal migration: complementary roles for transendothelial migration}

UM cells treated with Arp2/3 complex inhibitor interacted with the endothelial monolayer and performed TEM, but the cells did not switch from the amoeboid mode to the mesenchymal mode of mo- tility and migration. In this case, the intercalation step was dispensable for TEM, and the amoeboid mode of behavior alone was sufficient. This finding suggests that UM cells in situ may use a combination of amoeboid and mesenchymal modes of migration, depending on the local circumstances, such as the architecture of the vascular bed and the flow of the bloodstream. One might speculate that inside-out signaling from Rac and PAK activates adhesion molecules on the UM cell surface, such as integrin $\alpha 4 \beta 1$ (Rullo et al., 2012), required for adhesion to endothelial cells (Onken et al., 2014a). This hypothesis is supported by our observation of decreased interactions of Rac/PAK-inhibited UM cells with the endothelial cell monolayer, compared with untreated or Arp2/3-inhibited UM cells.

Another possible explanation for the effect of Arp2/3 complex inhibition is a requirement for branched actin assembly to stabilize early interactions that promote the maturation of adhesive junctions. One model of cell-cell adhesion formation is that weak interactions at a small cell-cell contact surface recruit active Rac1, which activates actin assembly to expand the contact surface and form a mature adhesion (Collins and Nelson, 2015). This model is based on observations that Arp2/3 complex inhibition disrupts junction formation between cells on plastic but not the formation of cell aggregates (Collins and Nelson, 2015). Cells that aggregate with neighboring cells do not require extensive Arp2/3-dependent lamellipodia activity, but cells that are flat and spread out require expanded contacts through lamellipodia, which would require Arp2/3 activity (Collins et al., 2017). This model predicts that blocking branched actin formation by inhibiting Rac1 or PAK would also prevent UM cells from establishing sufficiently strong adhesions with the endothelial monolayer. Our results expand this view by suggesting that separate adhesive mechanisms exist, dependent on and independent of stabilization by actin assembly during TEM.

\section{Metastasis-promoting BAP1 mutation favors mesenchymal motility and increases transmigration}

We discovered that partial loss of BAP1, based on heterozygous gene deletion, caused increases in all steps of TEM. This discovery is important because in human UM patients, the loss of BAP1 is the key genetic feature associated with a high risk of metastasis in the class 2 lethal genotype (Harbour et al., 2010; Matatall et al., 2013). The clinical relationship of deletion of BAP1 to metastatic spread is consistent with the biological connection of BAP1 function with the mechanism of TEM discovered here. The genomic transcriptional targets of BAP1 include a number of actin cytoskeleton genes, including TRIO, cortactin, and various GAPs and GEFs (Figure S1A) (Yen et al., 2018). BAP1 targets of particular note include ARHGAP18, a Rho-specific GAP, and ARHGEF4, a Rac/cdc42-specific GEF (Yen et al., 2018), 


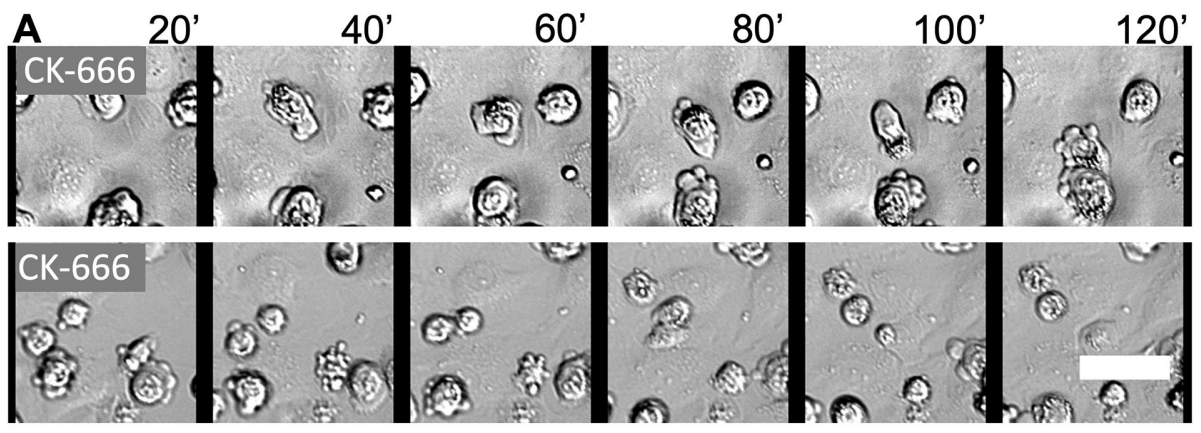

B
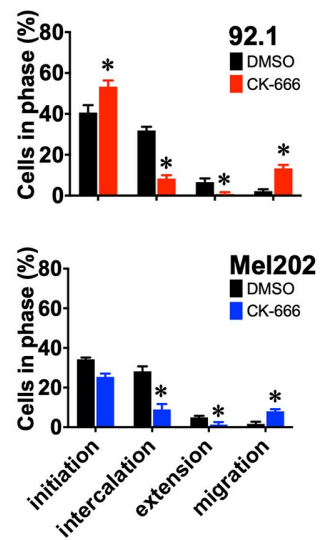

C
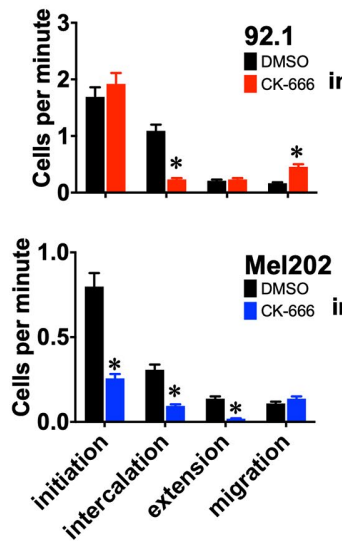

D

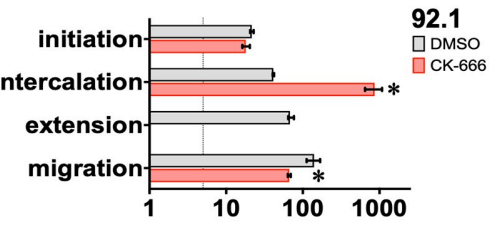

Mel202
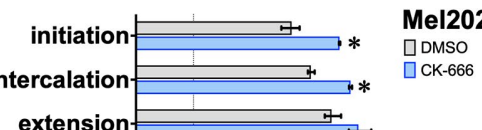

migration

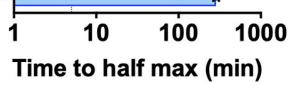

FIGURE 4: Effects of Arp2/3 complex inhibition on TEM of UM cells. (A) CK-666 blocked the switch to lamellipodial activity, decreasing intercalation (upper panels and Supplemental Video S4) and increasing direct transmigration bypassing intercalation (lower panels). (B) Endpoint values of cells that have undergone each step of transmigration based on results from three 2-h movies for each condition for each cell line. The total numbers of 92.1 cells scored were 321 (vehicle) and 360 (CK-666); the total numbers of Mel202 cells scored were 365 (vehicle) and 632 (CK-666). (C) Maximum rates were decreased for intercalation but increased for migration. (D) Times to reach half of the calculated maximum plateaus. Scale bar, $50 \mu \mathrm{m} ;{ }^{*} p<0.01$.

Cell lines, plasmids, and viruses

Cells were cultured at $37^{\circ} \mathrm{C}$ in a humidified $5 \% \mathrm{CO}_{2}$ incubator. Human $92.1 \mathrm{UM}$ cells (RRID: CVCL_8607) were the generous gift of Martine Jager (Laboratory of Ophthalmology, Leiden University, Netherlands) and were grown in RPMI 1640 medium (Life Technologies, Carlsbad, CA) supplemented with $10 \%$ FBS and antibiotics. Primary human dermal microvascular endothelial cells (HDMVECs: HMVEC-dNeo, Catalog \#CC2516; Lonza, Allendale, NJ) were grown in EGM-2 MV culture medium (Lonza). HDMVECs were not used after eight passages. BAP1(+/-) 92.1 subclone cell lines were generated using CRISPR/Cas9 genome editing and sequence-verified by the Washington University Genome Engineering and iPSC Center (http://geic.wustl.edu). Briefly, single guide RNAs were used to target Cas9 to exon 3 of BAP1 in 92.1 cells to generate frameshifted truncation mutants. The 1D7 strain is heterozygous for a 53-bp deletion: p.V24fsX26 (c.183-237 delGTCAAGGGGGTGCAAGTGGAGGAGATCTACGACCTTCAGAGCAAATGTCAGGG) (Supplemental Figure S4). The 2D3 strain is heterozygous for a 43-bp deletion: p.K25fsX32 (c. 186-230 delAAGGGGGTGC AAGTGGAGGAGATCTACGACCTTCAGAGCAAAT; Supplemental Figure S4).

Lentiviral-based constructs were used for cell expression experiments. The pSLIK-DNRhoA(T19N) dominant-negative YFP-fusion expression construct was a gift from Sanjay Kumar (Addgene plasmid \#84646; RRID:Addgene_84646; MacKay and Kumar,

both of which are up-regulated in BAP1-deficient Class 2 human tumors (Onken et al., 2004; Supplemental Figure S1B). Concurrent up-regulation of ARHGAP18 and ARHGEF4 would be expected to decrease RhoA signaling and increase Rac1 signaling and promote TEM. In support of this hypothesis, increased expression of ARHGAP18 (Maeda et al., 2011; Humphries et al., 2017) and ARHGEF4 (Taniuchi et al., 2018) has been linked to metastasis in other cancers.

\section{MATERIALS AND METHODS}

Request a protocol through Bio-protocol.

\section{Reagents and inhibitors}

Chemicals and reagents were obtained from Fisher Scientific (Pittsburgh, PA) or Sigma-Aldrich (Saint Louis, MO), except as follows: Blebbistatin, Y-27632, NSC-23766, and IPA-3 were from Selleck Chemicals (Houston, TX). FR900359 was prepared as described by Onken et al. (2018). Inhibitors were dissolved in DMSO as stock solutions and frozen in small aliquots. For TEM experiments, Blebbistatin and Y-27632 were used at $10 \mu \mathrm{M}$, IPA-3 was used at $30 \mu \mathrm{M}, \mathrm{CK}-666$ was used at $100 \mu \mathrm{M}$, and FR900359 was used at $100 \mathrm{nM}$. Inhibitors were tested for cell toxicity using a tetrazolium-based Cell Counting Kit-8 (Bimake, Houston, TX), with treatment at working concentrations for $12 \mathrm{~h}$. In these assays, the inhibitors did not significantly reduce cell viability, with the exception of FR900359, which has longterm effects that have been described elsewhere (Onken et al., 2018).
2014). Viral production and infections were carried out according to consortium recommendations (https://www.broadinstitute.org/rnaiconsortium/) using HEK293T cells transfected with third-generation lentivirus packaging plasmids, pMDLg/pRRE, pRSV-Rev, and PMD2.G, using TransIT-LT1 transfection reagent (MirusBio, Wisconsin). Lentivirus supernatants were filtered with a $0.45-\mu \mathrm{m}$ pore filter (EMD Millipore Corp, USA) and concentrated at 20,000 rpm for $2 \mathrm{~h}$. Lentiviral particles were resuspended and stored in aliquots at $-70^{\circ} \mathrm{C}$. For protein expression, lentivirus was added directly to UM cells grown in six-well plates containing $8 \mu \mathrm{g} / \mathrm{ml}$ protamine sulfate (Sigma-Aldrich). After $36 \mathrm{~h}$, the medium was changed to growth medium containing $200 \mathrm{ng} / \mathrm{ml}$ doxycycline hydrochloride (Alfa Aesar, Ward Hill, MA) and incubated an additional $48 \mathrm{~h}$ to induce expression. Fusion protein expression was determined by presence of YFP during live-cell imaging. The pcDNA3-GFP-Rac1(T17N) dominant negative expression construct was purchased from Cell Biolabs (San Diego, CA, cat\# STA-450) and transfected directly into 92.1 and Mel202 cells using TransIT-LT1 transfection reagent (MirusBio, Wisconsin). Fusion protein expression was determined by the presence of GFP during live-cell imaging.

\section{Imaging of live cells: transendothelial migration}

Polyacrylamide hydrogels were prepared with $0.4 \%$ bis-acrylamide on glass-bottomed microwell dishes (MatTek, Ashland, MA) as described by Onken et al. (2014b). The hydrogels were coated with 

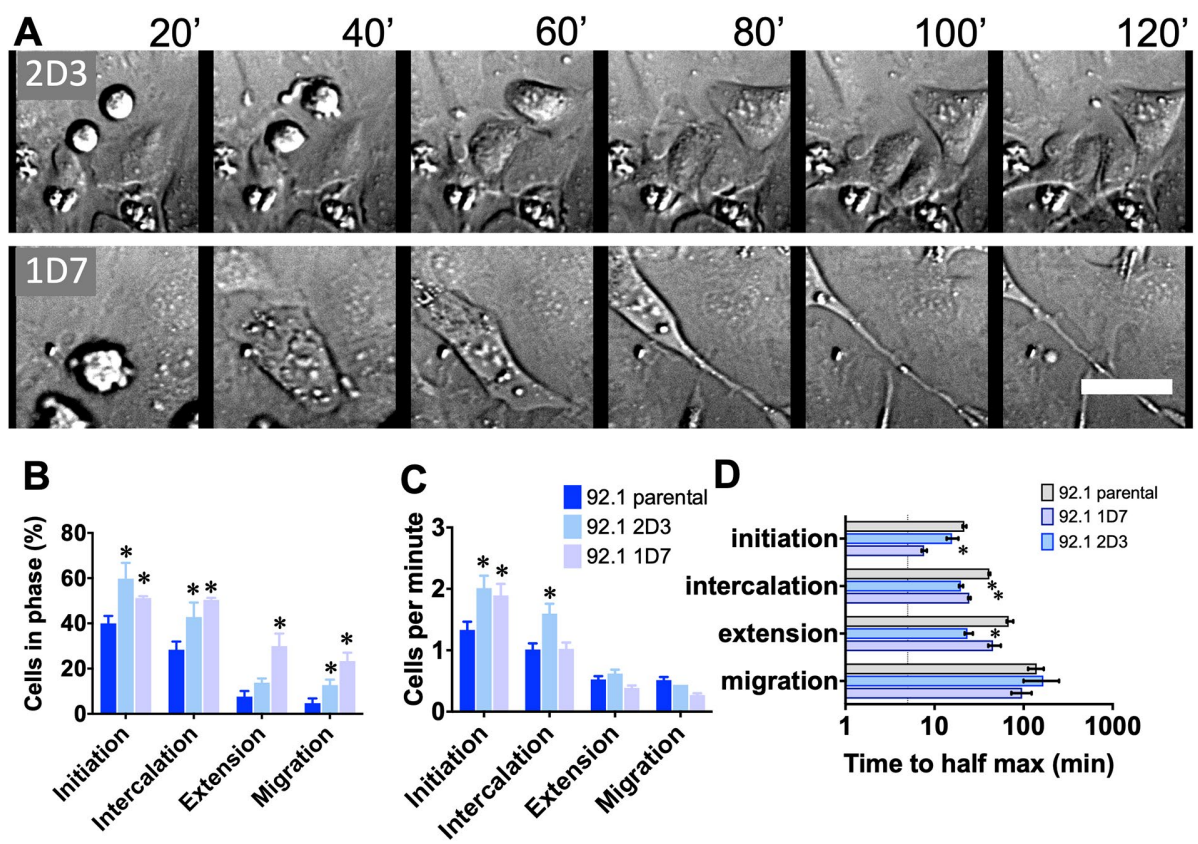

D

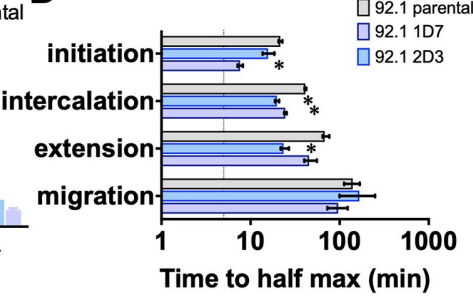

FIGURE 5: BAP1-deficient UM cells show increases in all steps of TEM. (A) Frames from time-lapse movies of two independent BAP1(+/-) subclones of the UM 92.1 cell line: 2D3 (upper panels and Supplemental Video S5) and 1D7 (lower panels). (B) Endpoint values of cells that have undergone each step based on results from three 2-h movies for each cell line. The total numbers of cells scored were 395 (parental), 253 (2D3), and 203 (1D7). (C) Maximum rates for each step. (D) Times to reach half of the calculated maximum plateaus. Scale bar, $50 \mu \mathrm{m}$; ${ }^{*} p<0.01$.

fibronectin $\left(10 \mu \mathrm{g} / \mathrm{ml}\right.$ in PBS) by incubation at $37^{\circ} \mathrm{C}$ for $30 \mathrm{~min}$. HDMVECs were plated onto the fibronectin-coated hydrogel substrates and incubated overnight to allow formation of monolayers.

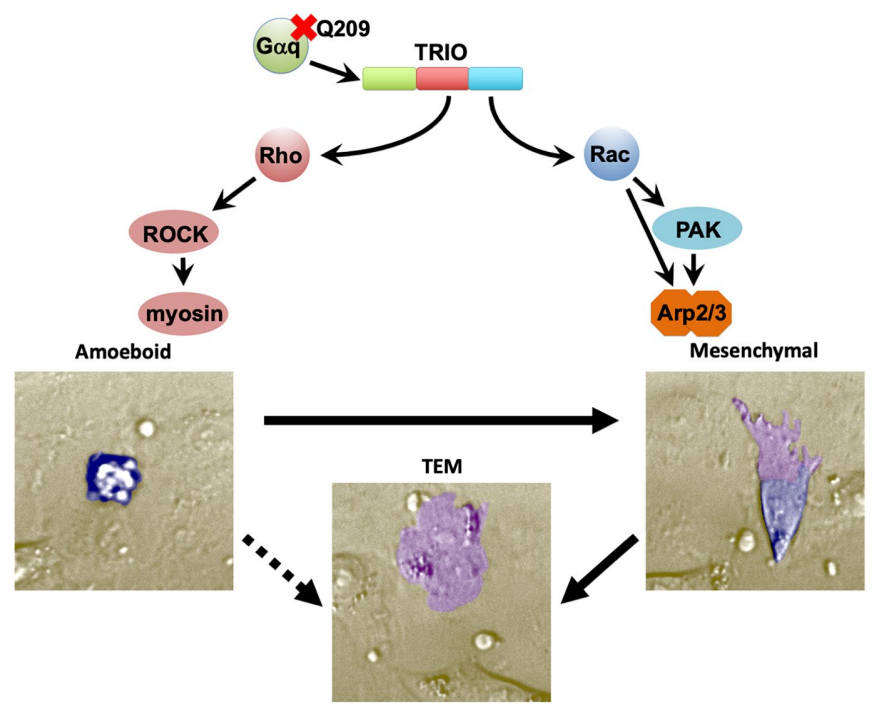

FIGURE 6: Diagram summarizing UM cell signaling and modes of motility during TEM. Constitutively active $\mathrm{Gq}$, the oncogenic driver for UM, signals through the dual-GEF TRIO, which activates both RhoA and Rac1. Rho signals through ROCK to myosin-Il, which promotes amoeboid motility. Rac activates PAK and Arp2/3, which promote lamellar and lamellipodial protrusions as part of mesenchymal motility. The switch from amoeboid to mesenchymal motility increases the robustness of TEM, but TEM can occur via a solely amoeboid mechanism.
HDMVEC monolayers were allowed to mature for 3 days before their use in TEM assays. Monolayers were inspected by phasecontrast microscopy to ensure that cells covered the substrate completely, without defects, before transmigration assays were performed, as previously described (Onken et al., 2014a,b). Monolayers were rinsed with fresh EGM-2 MV media to remove floating cells and debris $1 \mathrm{~h}$ before assaying TEM.

UM cells were trypsinized, collected in medium containing FBS to stop tryptic activity, and then centrifuged and resuspended in EGM-2 MV culture medium at $10^{5} \mathrm{cell} / \mathrm{ml}$. Inhibitors or vehicle were added to individual aliquots of UM cells $2 \mathrm{~h}$ before TEM imaging, except for FR900359, which was added to stock plates 1 day before imaging ( $>24 \mathrm{~h}$ ) and again after harvesting and resuspension in EGM-2 MV. For imaging, HDMVEC monolayers were placed in an environmental chamber (Stage Top Incubator, Tokai Hit, Shizuoka-ken, Japan) with $5 \% \mathrm{CO}_{2}$ at $37^{\circ} \mathrm{C}$ on an inverted microscope (Olympus IX72) and reassessed visually by DIC to identify fields for imaging that were absent of defects and showed visible junctions between all endothelial cells.

After monolayer integrity was confirmed, $10^{4}$ UM cells were added to the monolayer directly over the objective. DIC images were captured at 20-s intervals with a $10 \times$ objective. Scoring of TEM events was performed as described by Onken et al. (2014a). In DIC images, UM cells are round and bright when added to the monolayers (for example, see Supplemental Figure S3, 5'). UM cells that interact with the top of the monolayer remain bright, even as they bleb and change shape (Supplemental Figure S3, 27'). At the initiation of transmigration, UM cells send protrusions between cells in the endothelial monolayer, and the distal portions of these protrusions become visible beneath the endothelial cells as flat, fanlike projections in DIC images (Supplemental Figure S3, 32'). The first frame with visible projections is scored as the time of "initiation" for the associated UM cell. The transmigrating UM cells then expand the opening in the endothelial junction and fill the space between the endothelial cells by flattening and becoming larger and less bright, resembling the adjacent endothelial cells in DIC (Supplemental Figure S3, 50'). The first frame in which the UM cell morphology stops changing and the cell settles among endothelial cells is scored as "intercalation." Later, intercalated UM cells send new protrusions that are visible as large flattened projections beneath the endothelial cells (Supplemental Figure S3, 89'); the first frame in which this projection is visible is scored as "extension." Some UM cells proceed to extend this projection underneath the monolayer, expanding and elongating the flattened protrusion. They release their adhesions to the endothelial cells opposite the protrusion and pull their cell body and nucleus beneath the monolayer, in the direction of the leading protrusion (Supplemental Figure S3, 102 '). The first frame in which the UM cell nucleus is completely beneath the monolayer is scored as "migration" (Supplemental Figure S3, 108'). In the case of treatment with CK-666, UM cells that transmigrated directly beneath the monolayer, without intercalating, were only scored for "initiation" and "migration." 
For dominant-negative expression experiments, epifluorescence images were collected to detect cells expressing YFP (RhoA-DN) or GFP (Rac1-DN) and only positive cells were analyzed by DIC imaging. To control for biological variability among different lots of primary human endothelial cells, DMSO-treated experiments were performed with every inhibitor experiment, and data for each experimental condition were collected from three separate controlled experiments.

\section{Quantification and statistical analysis}

Statistical analyses were performed with Prism (GraphPad, San Diego, CA). For endpoint cell counts, the mean and standard error of the mean were calculated from at least three experiments for each inhibitor, and statistical analysis compared each inhibitor experiment with control experiments from the same day. To generate cumulative percentage curves, data from all experiments for a given inhibitor were combined, and these curves were used to quantify rates. Nonlinear regression was used to fit curves for each step, which extrapolated to maximal completion plateau values used to quantify time to half maximal completion. To calculate maximum rates, tangent lines were drawn either at the most vertical points of curves that showed clear change over time or along the longest linear stretch for curves that followed a more linear course. Times and rates for each step were normalized to values generated from control experiments run on the same day and $90 \%$ confidence intervals were calculated.

\section{ACKNOWLEDGMENTS}

This work was supported by NIH Grants GM118171 to J.A.C. and CA234533 to K.J.B.

\section{REFERENCES}

Acton SE, Farrugia AJ, Astarita JL, Mourão-Sá D, Jenkins RP, Nye E, Hooper S, van Blijswijk J, Rogers NC, Snelgrove KJ, et al. (2014). Dendritic cells control fibroblastic reticular network tension and lymph node expansion. Nature 514, 498-502.

Anand K, Roszik J, Gombos D, Upshaw J, Sarli V, Meas S, Lucci A, Hall C, Patel S (2019). Pilot study of circulating tumor cells in early-stage and metastatic uveal melanoma. Cancers (Basel) 11, doi:10.3390/cancers11060856

Bokoch GM (2003). Biology of the p21-activated kinases. Annu Rev Biochem 72, 743-781.

Byrne KM, Monsefi N, Dawson JC, Degasperi A, Bukowski-Wills JC, Volinsky N, Dobrzy ski M, Birtwistle MR, Tsyganov MA, Kiyatkin A, et al. (2016). Bistability in the Rac1, PAK, and RhoA signaling network drives actin cytoskeleton dynamics and cell motility switches. Cell Syst 2, 38-48.

Callejo SA, Antecka E, Blanco PL, Edelstein C, Burnier MN (2006). Identification of circulating malignant cells and its correlation with prognostic factors and treatment in uveal melanoma. A prospective longitudinal study. Eye 21, 752-759.

Carvajal RD, Schwartz GK, Tezel T, Marr B, Francis JH, Nathan PD (2017). Metastatic disease from uveal melanoma: treatment options and future prospects. Br J Ophthalmol 101, 38-44.

Chrzanowska-Wodnicka M, Burridge K (1996). Rho-stimulated contractility drives the formation of stress fibers and focal adhesions. J Cell Biol 133, $1403-1415$

Clarijs R, Otte-Holler I, Ruiter DJ, de Waal RM (2002). Presence of a fluidconducting meshwork in xenografted cutaneous and primary human uveal melanoma. Invest Ophthalmol Vis Sci 43, 912-918.

Clarijs R, Schalkwijk L, Ruiter DJ, de Waal RM (2001). Lack of lymphangiogenesis despite coexpression of VEGF-C and its receptor Flt-4 in uveal melanoma. Invest Ophthalmol Vis Sci 42, 1422-1428.

Collins C, Denisin AK, Pruitt BL, Nelson WJ (2017). Changes in E-cadherin rigidity sensing regulate cell adhesion. Proc Natl Acad Sci USA 114, E5835-E5844.

Collins C, Nelson WJ (2015). Running with neighbors: coordinating cell migration and cell-cell adhesion. Curr Opin Cell Biol 36, 62-70.
Cooper S, Sadok A, Bousgouni V, Bakal C (2015). Apolar and polar transitions drive the conversion between amoeboid and mesenchymal shapes in melanoma cells. Mol Biol Cell 26, 4163-4170.

Dey A, Seshasayee D, Noubade R, French DM, Liu J, Chaurushiya MS, Kirkpatrick DS, Pham VC, Lill JR, Bakalarski CE, et al. (2012). Loss of the tumor suppressor BAP1 causes myeloid transformation. Science 337, $1541-1546$.

Feng X, Arang N, Rigiracciolo DC, Lee JS, Yeerna H, Wang Z, Lubrano S, Kishore A, Pachter JA, König GM, et al. (2019). A platform of synthetic lethal gene interaction networks reveals that the GNAQ uveal melanoma oncogene controls the hippo pathway through FAK. Cancer Cell 35, 457-472.e5.

Feng $X$, Degese MS, Iglesias-Bartolome R, Vaque JP, Molinolo AA, Rodrigues M, Zaidi MR, Ksander BR, Merlino G, Sodhi A, et al. (2014). Hippo-independent activation of YAP by the GNAQ uveal melanoma oncogene through a trio-regulated rho GTPase signaling circuitry. Cancer Cell 25, 831-845.

Friedl P, Wolf K (2003). Proteolytic and non-proteolytic migration of tumour cells and leucocytes. Biochem Soc Symp 277-285. doi:10.1042/ bss0700277

Guilluy C, Garcia-Mata R, Burridge K (2011). Rho protein crosstalk: another social network. Trends Cell Biol 21, 718-726.

Haga RB, Ridley AJ (2016). Rho GTPases: regulation and roles in cancer cell biology. Small GTPases 7, 207-221.

Harbour JW, Onken MD, Roberson ED, Duan S, Cao L, Worley LA, Council ML, Matatall KA, Helms C, Bowcock AM (2010). Frequent mutation of BAP1 in metastasizing uveal melanomas. Science 330 1410-1413.

Hart T, Chandrashekhar M, Aregger M, Steinhart Z, Brown KR, MacLeod G, Mis M, Zimmermann M, Fradet-Turcotte A, Sun S, et al. (2015). Highresolution CRISPR screens reveal fitness genes and genotype-specific cancer liabilities. Cell 163, 1515-1526.

Humphries B, Wang Z, Li Y, Jhan JR, Jiang Y, Yang C (2017). ARHGAP18 downregulation by miR-200b suppresses metastasis of triple-negative breast cancer by enhancing activation of RhoA. Cancer Res 77, 4051-4064

Keilholz U, Goldin-Lang P, Bechrakis NE, Max N, Letsch A, Schmittel A, Scheibenbogen C, Heufelder K, Eggermont A, Thiel E (2004). Quantitative detection of circulating tumor cells in cutaneous and ocular melanoma and quality assessment by real-time reverse transcriptasepolymerase chain reaction. Clin Cancer Res 10, 1605-1612.

Krantz BA, Dave N, Komatsubara KM, Marr BP, Carvajal RD (2017). Uveal melanoma: epidemiology, etiology, and treatment of primary disease. Clin Ophthalmol 11, 279-289.

MacKay JL, Kumar S (2014). Simultaneous and independent tuning of RhoA and Rac1 activity with orthogonally inducible promoters. Integr Biol (Camb) 6, 885-894.

Maeda M, Hasegawa H, Hyodo T, Ito S, Asano E, Yuang H, Funasaka K, Shimokata K, Hasegawa Y, Hamaguchi M, Senga T (2011). ARHGAP18, a GTPase-activating protein for RhoA, controls cell shape, spreading, and motility. Mol Biol Cell 22, 3840-3852.

Matatall KA, Agapova OA, Onken MD, Worley LA, Bowcock AM, Harbour JW (2013). BAP1 deficiency causes loss of melanocytic cell identity in uveal melanoma. BMC Cancer 13, 371.

Mihic-Probst D, Ikenberg K, Tinguely M, Schraml P, Behnke S, Seifert B, Civenni G, Sommer L, Moch H, Dummer R (2012). Tumor cell plasticity and angiogenesis in human melanomas. PLoS One 7, e33571.

Moore AR, Ceraudo E, Sher JJ, Guan Y, Shoushtari AN, Chang MT, Zhang JQ, Walczak EG, Kazmi MA, Taylor BS, Huber T, et al. (2016). Recurrent activating mutations of G-protein-coupled receptor CYSLTR2 in uveal melanoma. Nat Genet 48, 675-680.

Nobes CD, Hall A (1995). Rho, rac, and cdc42 GTPases regulate the assembly of multimolecular focal complexes associated with actin stress fibers, lamellipodia, and filopodia. Cell 81, 53-62.

Nobes CD, Hall A (1999). Rho GTPases control polarity, protrusion, and adhesion during cell movement. J Cell Biol 144, 1235-1244.

Nolen BJ, Tomasevic N, Russell A, Pierce DW, Jia Z, McCormick CD, Hartman J, Sakowicz R, Pollard TD (2009). Characterization of two classes of small molecule inhibitors of Arp2/3 complex. Nature 460, 1031-1034.

Onken MD, Li J, Cooper JA (2014a). Uveal melanoma cells utilize a novel route for transendothelial migration. PLoS One 9, e115472.

Onken MD, Makepeace CM, Kaltenbronn KM, Kanai SM, Todd TD, Wang S, Broekelmann TJ, Rao PK, Cooper JA, Blumer KJ (2018). Targeting nucleotide exchange to inhibit constitutively active $\mathrm{G}$ protein $\alpha$ subunits in cancer cells. Sci Signal. 11, doi:10.1126/scisignal.aao6852 
Onken MD, Mooren OL, Mukherjee S, Shahan ST, Li J, Cooper JA (2014b). Endothelial monolayers and transendothelial migration depend on mechanical properties of the substrate. Cytoskeleton (Hoboken) 71, 695-706.

Onken MD, Worley LA, Ehlers JP, Harbour JW (2004). Gene expression profiling in uveal melanoma reveals two molecular classes and predicts metastatic death. Cancer Res 64, 7205-7209.

Parri M, Chiarugi P (2010). Rac and Rho GTPases in cancer cell motility control. Cell Commun Signal 8, 23.

Rullo J, Becker H, Hyduk SJ, Wong JC, Digby G, Arora PD, Cano AP, Hartwig J, McCulloch CA, Cybulsky MI (2012). Actin polymerization stabilizes $\alpha 4 \beta 1$ integrin anchors that mediate monocyte adhesion. J Cell Biol 197, 115-129.

Sahai E, Marshall CJ (2003). Differing modes of tumour cell invasion have distinct requirements for Rho/ROCK signalling and extracellular proteolysis. Nat Cell Biol 5, 711-719.

Sander EE, ten Klooster JP, van Delft S, van der Kammen RA, Collard JG (1999). Rac downregulates Rho activity: reciprocal balance between both GTPases determines cellular morphology and migratory behavior. J Cell Biol 147, 1009-1022.

Sanz-Moreno V, Gadea G, Ahn J, Paterson H, Marra P, Pinner S, Sahai E, Marshall CJ (2008). Rac activation and inactivation control plasticity of tumor cell movement. Cell 135, 510-523.

Sanz-Moreno V, Gaggioli C, Yeo M, Albrengues J, Wallberg F, Viros A, Hooper S, Mitter R, Féral CC, Cook M, et al. (2011). ROCK and JAK1 signaling cooperate to control actomyosin contractility in tumor cells and stroma. Cancer Cell 20, 229-245.

Schmidt S, Debant A (2014). Function and regulation of the Rho guanine nucleotide exchange factor Trio. Small GTPases 5, e29769.
Taniuchi K, Furihata M, Naganuma S, Saibara T (2018). ARHGEF4 predicts poor prognosis and promotes cell invasion by influencing ERK1/2 and GSK-3 $\alpha / \beta$ signaling in pancreatic cancer. Int J Oncol 53, 2224-2240.

Testa JR, Cheung M, Pei J, Below JE, Tan Y, Sementino E, Cox NJ, Dogan AU, Pass HI, Trusa S, et al. (2011). Germline BAP1 mutations predispose to malignant mesothelioma. Nat Genet 43, 1022-1025.

Urtatiz O, Cook C, Huang JL, Yeh I, Van Raamsdonk CD (2020). GNAQ ${ }^{\text {Q209L }}$ expression initiated in multipotent neural crest cells drives aggressive melanoma of the central nervous system. Pigment Cell Melanoma Res 33, 96-111.

van der Kooij MK, Speetjens FM, van der Burg SH, Kapiteijn E (2019). Uveal versus cutaneous melanoma; same origin, very distinct tumor types. Cancers (Basel) 11, doi:10.3390/cancers11060845

Van Raamsdonk CD, Bezrookove V, Green G, Bauer J, Gaugler L, O'Brien JM, Simpson EM, Barsh GS, Bastian BC (2009). Frequent somatic mutations of GNAQ in uveal melanoma and blue naevi. Nature 457 599-602.

Van Raamsdonk CD, Griewank KG, Crosby MB, Garrido MC, Vemula S, Wiesner T, Obenauf AC, Wackernagel W, Green G, Bouvier N, et al. (2010). Mutations in GNA11 in uveal melanoma. N Engl J Med doi:10.1056/ NEJMoa1000584

Wyckoff JB, Pinner SE, Gschmeissner S, Condeelis JS, Sahai E (2006). ROCK- and myosin-dependent matrix deformation enables proteaseindependent tumor-cell invasion in vivo. Curr Biol 16, 1515-1523.

Yen M, Qi Z, Chen X, Cooper JA, Mitra RD, Onken MD (2018). Transposase mapping identifies the genomic targets of BAP1 in uveal melanoma. BMC Med Genomics 11, 97.

Zhao ZS, Manser E (2012). PAK family kinases: Physiological roles and regulation. Cell Logist 2, 59-68. 